\title{
SEVERE RIGHT VENTRICULAR FAILURE FOLLOWING PERICARDIOCENTESIS: A CASE REPORT OF PERICARDIAL DECOMPRESSION SYNDROME
}

Sergio Perez ${ }^{1}$, Jennifer Amastha ${ }^{1}$, Louis Vincent ${ }^{1}$, Carlos Alfonso ${ }^{2}$, and Eduardo de Marchena ${ }^{3}$

${ }^{1}$ University of Miami

${ }^{2}$ University of Miami, Miller School of Medicine

${ }^{3}$ University of Miami Miller School of Medicine

August 11, 2020

\begin{abstract}
Pericardial decompression syndrome is a rare but potentially fatal complication following needle or surgical pericardiocentesis and should be recognized as paradoxical hemodynamic deterioration. The exact pathophysiology of pericardial decompression syndrome is unknown but is likely that several mechanisms involving hemodynamic, ischemic and autonomic imbalance lead to the clinical manifestation of this entity. There is no specific treatment for pericardial decompression syndrome other than supportive care, however, early interventions such as aggressive heart failure therapy, inotropic medications and sometimes mechanical circulatory support should be implement as mortality can be as high as $30 \%$. We report a patient presenting with severe right ventricular failure and cardiogenic shock secondary to PDS.
\end{abstract}

SEVERE RIGHT VENTRICULAR FAILURE FOLLOWING PERICARDIOCENTESIS: A CASE REPORT OF PERICARDIAL DECOMPRESSION SYNDROME

Sergio A Perez ${ }^{\mathrm{a}}$, MD; Jennifer Amastha ${ }^{\mathrm{a}}$ MD; Louis Vincent ${ }^{\mathrm{a}}$, MD; Carlos E Alfonso ${ }^{\mathrm{a}}$, MD; Eduardo de Marchena $^{\mathrm{a}}, \mathrm{MD}$

${ }^{\text {a }}$ University of Miami, Division of Cardiovascular Medicine, University of Miami Hospital, Miami, Florida.

Total word count: 1570

Funding: This research received no specific grant from anyfunding agency in the public, commercial, or not-for-profit sectors

This study does not include any HIPPA patient identifier information. IRB approval and written informed consent were not required.

Disclosures: All authors have reported having no relationships relevant to the contents of this paper to disclose.

Address for Correspondence: Sergio A Perez, MD; 1400 NW 10th Avenue Dominion Tower Suite 206A. Miami Florida 33136. Phone 305-987-7915. Email: Sergio.andres.perez@gmail.com

ABSTRACT.

Pericardial decompression syndrome is a rare but potentially fatal complication following needle or surgical pericardiocentesis and should be recognized as paradoxical hemodynamic deterioration. The exact patho- 
physiology is unknown but is likely that several mechanisms involving hemodynamic, ischemic and autonomic imbalance are involved. There is no specific treatment other than supportive care, however, early interventions such as aggressive heart failure therapy, inotropic medications and sometimes mechanical circulatory support should be implemented as mortality can be as high as $30 \%$. We report a patient presenting with right ventricular failure and cardiogenic shock secondary to pericardial decompression syndrome.

\section{INTRODUCTION}

Pericardiocentesis is a lifesaving therapeutic procedure for patients presenting with cardiac tamponade. It is a relatively safe procedure however physicians must be aware of potential post-procedural complications. The risk ranges from $4 \%$ to $10 \%$, the most common being arrhythmias, coronary artery or cardiac puncture, pneumothorax and pneumopericardium [1]. Pericardial decompression syndrome (PDS) is a rare but potentially fatal complication after pericardiocentesis defined as paradoxical hypotension often with pulmonary edema and ventricular dysfunction following fluid drainage in patients with cardiac tamponade. Early recognition and aggressive initiation of supportive measures are essential. Morbidity and mortality remain elevated. Here, we report a case with an ominous outcome of profound cardiogenic shock after percutaneous drainage of a large pericardial effusion causing cardiac tamponade.

\section{CASE PRESENTATION}

73-year old woman presents to the hospital with 5-days of worsening dyspnea and fatigue. Examination was noticeable for heart rate of 110 beats per minute, blood pressure of $90 / 50 \mathrm{mmHg}$, jugular venous distention and pulsus paradoxus. Computed Tomography (CT) scan showed multiple hepatic and pulmonary lesions; enlarged mediastinal and abdominal lymph nodes and pleural and pericardial effusions concerning for metastatic cancer of unknown primary origin (Figure 1). She had elevated serum tumor markers including CA 19-9 and alpha feto-protein. An echocardiogram showed a large pericardial effusion with right ventricle (RV) diastolic collapse and 25-30\% respiratory variation in Doppler mitral inflow concerning for cardiac tamponade (Figure 2, Video 1).

She was taken to the catheterization laboratory for emergent needle pericardiocentesis. Approximately 750 $\mathrm{ml}$ of sanguineous fluid was drained and the patient was transferred to the ICU with the drain in place. She experienced brief improvement in her symptoms and the drain was removed 1 day later.

Repeat echocardiogram showed massive RV dilation and dysfunction and persistent ventricular interdependence (Figure 3 and 4). Electrocardiogram showed no changes suggestive of myocardial ischemia. On day 2, patient developed hypotension. On exam she had cold extremities with jugular venous distention. Laboratory showed metabolic acidosis with elevated lactic acid. Hemodynamics from bedside right heart catheterization using a Swan-Ganz catheter showed: right atrial pressure of $30 \mathrm{mmHg}$, right ventricular diastolic pressure of $31 \mathrm{mmHg}$, pulmonary artery pressure of $48 / 29 \mathrm{mmHg}$ and pulmonary capillary wedge pressure of $31 \mathrm{mmHg}$, consistent with right ventricular failure and elevated pulmonary capillary wedge pressure likely secondary to ventricular interdependence from RV enlargement. Cardiac output was $2.7 \mathrm{~L} / \mathrm{min}$ with a cardiac index of $1.7 \mathrm{~L} / \mathrm{min} / \mathrm{m}^{2}$. IV inotropic support was initiated with dobutamine. CT angiography demonstrated a small sub segmental pulmonary embolus in a right lower segmental branch. The pulmonary embolism was not felt to be large enough to explain the degree of right ventricular compromise.

She remained stable on dobutamine over the next 48 hours. Subsequently she acutely deteriorated with refractory hypotension, hypoxemia, lethargy and multi organ failure despite pharmacologic support. Echocardiogram showed persistent RV enlargement with abnormal septal motion (video 2-4). During emergent endotracheal intubation she had a pulseless electrical activity cardiac arrest. Despite resuscitative efforts she remained pulseless and was declared dead. Postmortem examination was declined by the family.

\section{DISCUSSION}

Our patient illustrates a case of PDS, a rare but potentially fatal complication after pericardiocentesis [2]. It is defined as worsening of hemodynamics after an uncomplicated pericardial drainage in patients with effusions and cardiac tamponade when hemodynamics are expected to improve. Other names used in the 
past include "low cardiac output syndrome" and "paradoxical hemodynamic instability" [3, 4]. Since the first description by Vandyke et al. in 1983 [5], other reports allowed wider recognition of this complication among clinicians.

The exact incidence of PDS is not precisely known given the wide variability in occurrence rates in different small case series but is estimated to be approximately $5 \%$. Clinical factors associated with an increased risk include history of malignancy or radiation therapy, preexisting cardiomyopathy with decreased systolic function and connective tissue disorders. In a study, surgical pericardiocentesis was the only variable associated with increased mortality when compared with needle pericardiocentesis in patients with PDS [6].

The onset of PDS after pericardial drainage is also variable. It can occur immediately after a brief initial improvement in patient's hemodynamics or within a couple of days and most commonly presents as acute congestive heart failure, often with pulmonary edema. In up to one third of the cases, PDS may present with development of cardiogenic shock.

The exact pathophysiology is not well understood but several mechanisms involving hemodynamic, ischemic and autonomic imbalance have been suggested. It is believed that right-sided chambers expansion resulting from increased venous return after removal of the compressing pericardial fluid can affect left ventricular filling and the effective cardiac output. Simultaneously the net increase in pulmonary venous return with an increased systemic vascular resistance can cause a preload/afterload mismatch and result in congestive heart failure. An additional contributing factor may be myocardial ischemia and stunning caused by impaired coronary artery perfusion, as it is known that increased intra-pericardial pressure affects maximal hyperemic coronary flow [7]. Lastly, the acute withdrawal of sympathetic stimulus after removal of effusion, has been hypothesized to provoke autonomic imbalance, which is theorized to occur either because of an unmasking of pre-existing myocardial dysfunction that was not apparent in the hyperadrenergic state with increased circulating catecholamines or because of induction of new myocardial dysfunction due to overwhelming autonomic stress via a mechanism similar to that of stress-induced cardiomyopathy. In fact, some have suggested that stress-induced cardiomyopathy and PDS may not be distinct entities but rather belong to the same spectrum of disease given the overlapping clinical presentation seen in some cases [8]. Our case however, lacks typical features of stress-induced cardiomyopathy: global RV involvement as opposed to apical ballooning with preserved basal function, no chest pain and no subsequent improvement of myocardial function.

There is no specific treatment for PDS other than supportive care. The exact mortality rate is not well known, but has been estimated to be around $30 \%$ based on case reports. Although ventricular dysfunction is transient and is expected to recover in survivors of PDS, patients will require advanced support measures such as aggressive heart failure therapy, inotropic medications and the use of mechanical circulatory support [9]. In cases of profound shock, the ideal type of mechanical support depends on the degree of myocardial dysfunction as well as the pattern of ventricular involvement, whether left, right or bi-ventricular dysfunction is noted.

Currently there are no proven measures known to prevent PDS. Despite the recommendation to drain fluid in $<1 \mathrm{~L}$ steps to avoid acute right-ventricular dilatation made by European Society of Cardiology 2004 guidelines [1], PDS may occur with drainage volumes $<500 \mathrm{ml}$. A reasonable strategy is to remove only enough fluid to alleviate tamponade physiology and slowly remove the remaining fluid by leaving the pericardial drain in place, especially in patients with cancer-related effusions or impressive chamber collapse.

\section{CONCLUSION.}

This uncommon case of PDS highlights the high morbidity and mortality associated with this complication, the possibility of instituting preventative strategies in high-risk cases, and the importance of prompt recognition of PDS and for close clinical monitoring and aggressive supportive care.

\section{REFERENCES}

1. Maisch, B., et al., Guidelines on the diagnosis and management of pericardial diseases executive summary; 
The Task force on the diagnosis and management of pericardial diseases of the European society of cardiology. Eur Heart J, 2004. 25 (7): p. 587-610.

2. Angouras, D.C. and T. Dosios, Pericardial decompression syndrome: a term for a well-defined but rather underreported complication of pericardial drainage. Ann Thorac Surg, 2010.89 (5): p. 1702-3; author reply 1703.

3. Dosios, T., et al., Thorough clinical investigation of low cardiac output syndrome after subxiphoid pericardiostomy. Angiology, 2007. 58 (4): p. 483-6.

4. Wagner, P.L., et al., Pericardial effusions in the cancer population: prognostic factors after pericardial window and the impact of paradoxical hemodynamic instability. J Thorac Cardiovasc Surg, 2011.141 (1): p. 34-8.

5. Vandyke, W.H., Jr., et al., Pulmonary edema after pericardiocentesis for cardiac tamponade. N Engl J Med, 1983.309 (10): p. 595-6.

6. Pradhan, R., et al., Patient characteristics and predictors of mortality associated with pericardial decompression syndrome: a comprehensive analysis of published cases. Eur Heart J Acute Cardiovasc Care, 2015. 4 (2): p. 113-20.

7. Skalidis, E.I., et al., Effect of pericardial pressure on human coronary circulation. Chest, 2000. 117 (3): p. 910-2.

8. Ayoub, C., M. Chang, and L. Kritharides, A case report of ventricular dysfunction post pericardiocentesis: stress cardiomyopathy or pericardial decompression syndrome? Cardiovasc Ultrasound, 2015.13 : p. 32.

9. Ricarte Bratti, J.P., et al., Venoarterial Extracorporeal Membrane Oxygenation Support for Severe Pericardial Decompression Syndrome: A Case Report. Crit Care Med, 2020.48 (1): p. e74-e75.

LEGENDS.

FIGURE 1. (A) CT scan showing a large pericardial effusion. (B) CT scan after pericardiocentesis showing significant RV enlargement (asterisk).

FIGURE 2. 2D-echocardiography four-chamber (A) and short-axis (B) views revealing massive pericardial effusion (asterisk)

FIGURE 3. 2D-echocardiography after pericardiocentesis. Four-chamber (A) and short-axis (B) views showing massive RV enlargement. Note the diameter of the RV (solid arrow) in comparison to that of the left ventricle (dashed arrow).

FIGURE 4. 2D-echocardiography after pericardiocentesis revealing decreased TAPSE of $6 \mathrm{~mm}$ on M-Mode (A) and S' velocity of $6 \mathrm{~cm} / \mathrm{sec}$ on tissue Doppler (B) consistent with RV dysfunction. Pulsed-wave Doppler $>25 \%$ mitral flow variation consistent with ventricular interdependence before (C) and after (D) pericardiocentesis. (TAPSE: tricuspid annular plane systolic excursion)

VIDEO 1. 2D-echocardiography, apical 4-chamber view revealing a large pericardial effusion

VIDEO 2. 2D-echocardiography, apical 4-chamber view

VIDEO 3. 2D-echocardiography, parasternal short axis view

VIDEO 4. 2D-echocardiography, apical 4-chamber view with agitated saline injection 

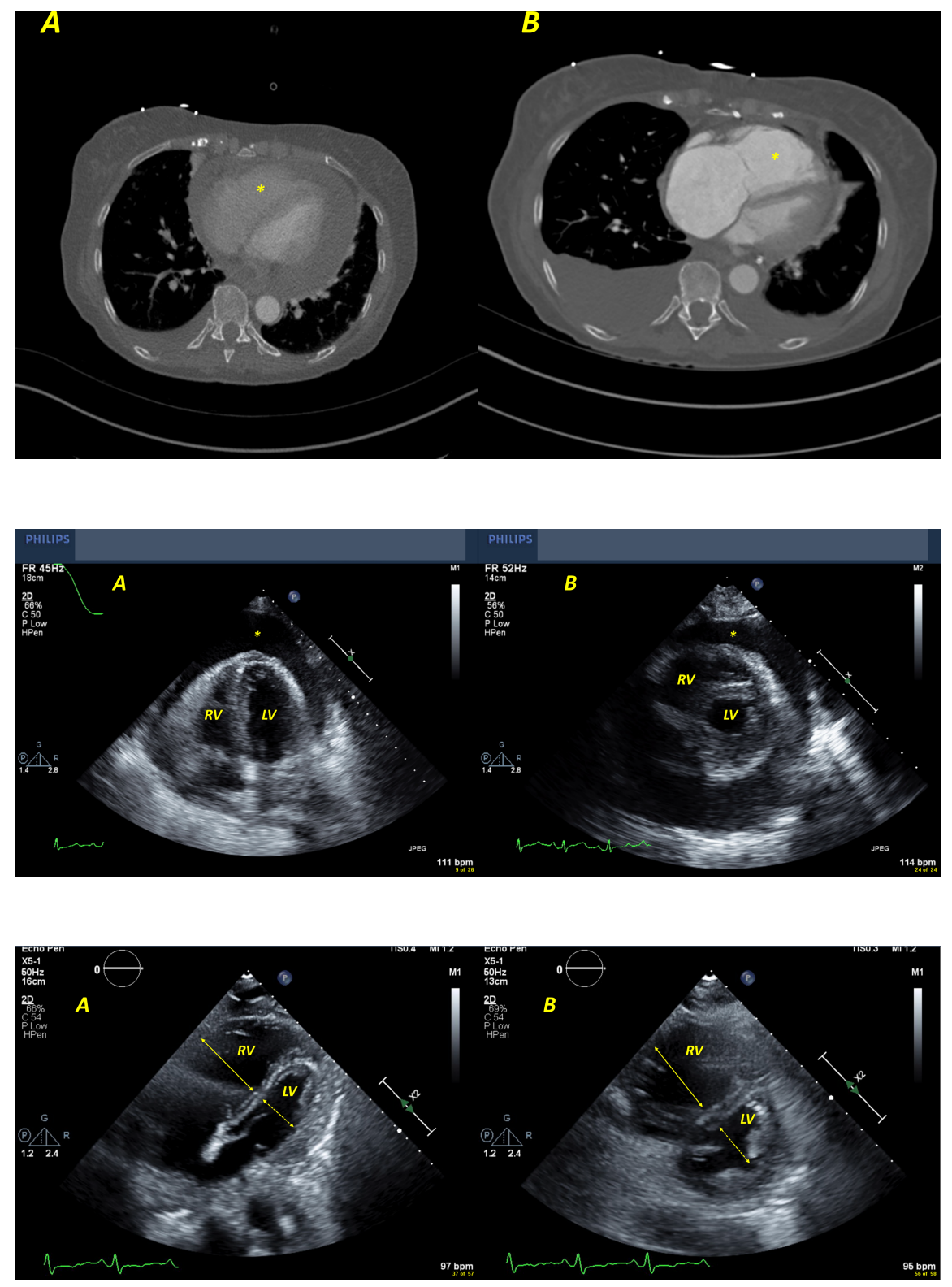


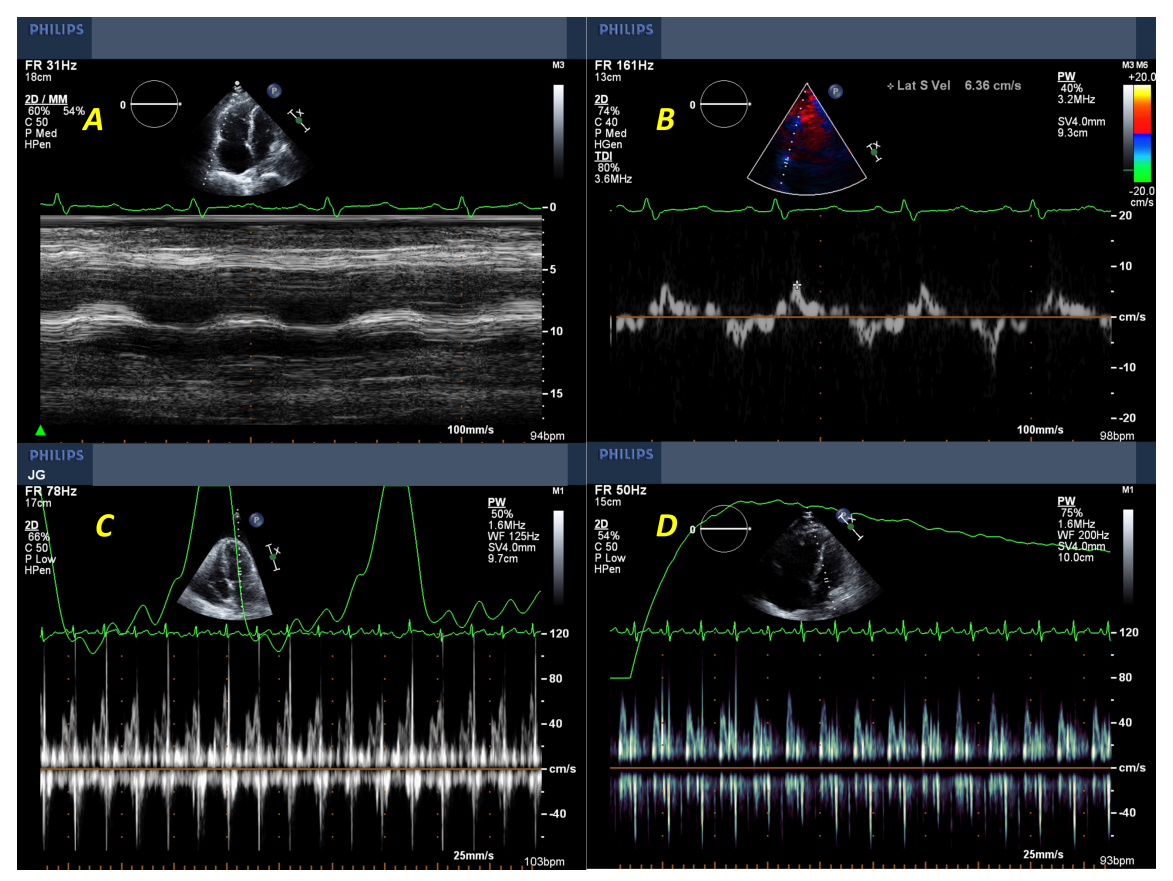

